\title{
Wave-Optics Analysis of Pupil Imaging
}

\author{
Bruce H. Dean, ${ }^{1}$ Brent J. Bos \\ Optics Branch / Code 551 \\ NASA Goddard Space Flight Center, Greenbelt, MD 20771
}

\begin{abstract}
Pupil imaging performance is analyzed from the perspective of physical optics. A multi-plane diffraction model is constructed by propagating the scalar electromagnetic field, surface by surface, along the optical path comprising the pupil imaging optical system. Modeling results are compared with pupil images collected in the laboratory. The experimental setup, although generic for pupil imaging systems in general, has application to the James Webb Space Telescope (JWST) optical system characterization where the pupil images are used as a constraint to the wavefront sensing and control process. Practical design considerations follow from the diffraction modeling which are discussed in the context of the JWST Observatory.
\end{abstract}

Keywords: wave optics, pupil imaging, diffraction analysis, wave front sensing, JWST

\section{INTRODUCTION}

A pupil imaging optical sub-system is designed to image the aperture stop of the parent optical system. The exit pupil (hereafter referred to as the "pupil") is defined as the image of the aperture stop as seen from the detector. In practice, the pupil image is an important diagnostic and systems engineering tool for assessing optical system performance. Factors affecting optical performance which are readily obtained from pupil image include: pupil geometry, obscuration profiles, radiometric data, and optical sub-system alignment. Obtaining this information from the pupil image will be limited by the pupil image quality which can be quantified and toleranced using conventional ray-trace techniques. But just as important in this assessment of image quality is predicting how image detail produced by the pupil imaging system will be affected by image ringing and other diffraction effects, recalling that diffraction occurs at the system stop, the object being imaged. Therefore, designing a pupil imaging system should not just include the standard ray-trace analysis, but also a multi-plane diffraction model. Modeling these effects falls under the domain of physical optics.

In this paper ${ }^{1}$ we discuss pupil imaging performance from the perspective of physical optics. In Section II, a waveoptics model is constructed by propagating the scalar electromagnetic field along the optical path comprising the pupil imaging system. In Section III, the model results are compared with pupil images collected in the laboratory. The experimental setup, although generic for pupil imaging systems in general, has application to the James Webb Space Telescope (JWST) ${ }^{2}$ optical system where the pupil images are used as a constraint to the wavefront sensing and control process. $^{3}$

\section{WAVE-OPTICS MODEL}

The optical system used for the analysis is illustrated in Fig. 1. The aperture stop in this system is the primary mirror. A wave-optics model is constructed by propagating the scalar electromagnetic field from surface to surface. For clarity, the PIL (pupil imaging lens) is excluded from Fig. 1 to emphasize that the complex amplitude of the scalar field at the focal plane of the mirror is given by the Fraunhofer propagation of the complex pupil, $A(x, y) e^{i k \varphi(x, y)}$ :

$$
u=\mathfrak{I}\left\{A(x, y) e^{i k \varphi(x, y)}\right\},
$$

where $(x, y)$ denote pupil coordinates and $k=2 \pi / \lambda$ for monochromatic imaging. The complex pupil function consists of the binary aperture function, $A(x, y)$, and a phase term, $\varphi(x, y)$, representing the optical path difference for the case of an aberrated mirror surface.

\footnotetext{
${ }^{1}$ Bruce.Dean@nasa.gov; phone: 1-301-286-8238; fax: 1-301-286-7204
} 


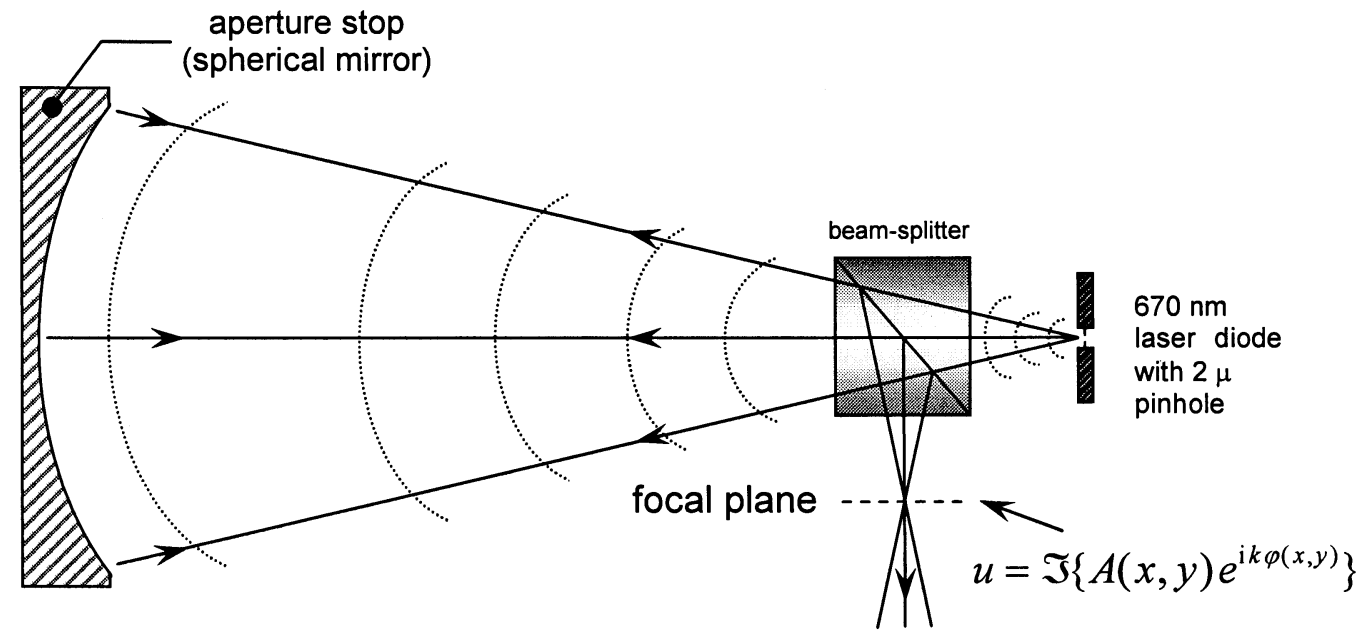

Fig. 1. Optical System

To find the field after propagation through the PIL, we begin first by specifying the field, $u_{1}$, immediately incident upon the first PIL surface 1 as illustrated in Fig. 2. For simplicity, the PIL is shown as a singlet in Fig. 2 but in implementation the PIL was actually a doublet from Edmund Scientific. ${ }^{4}$

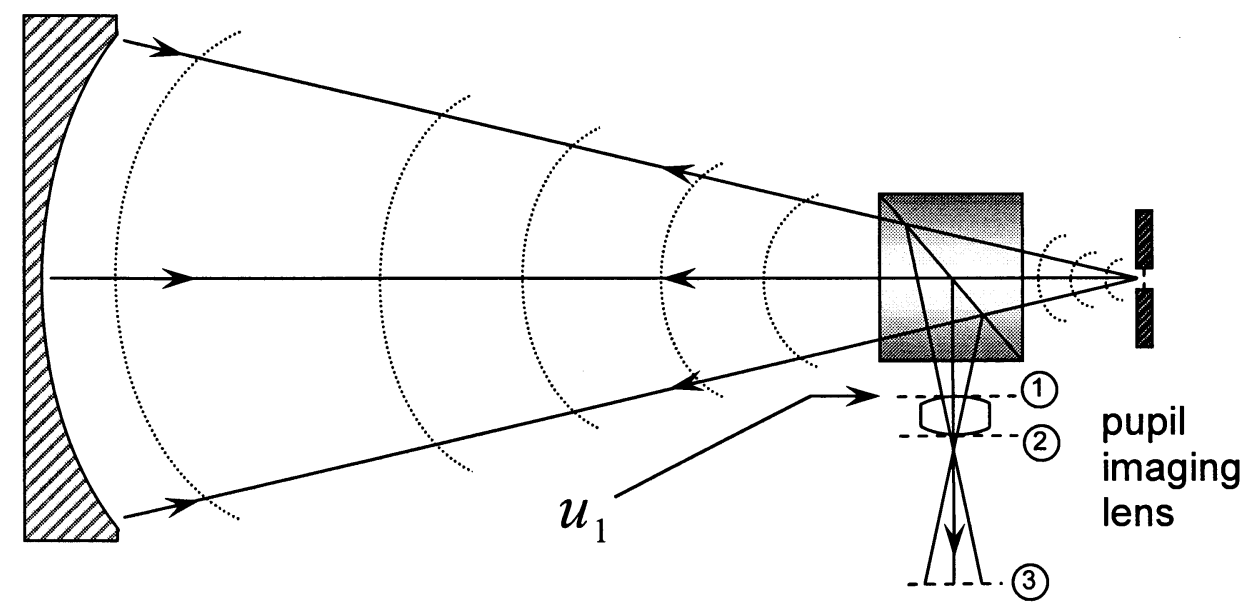

Fig. 2. Optical System with the Pupil Imaging Lens (PIL)

As shown in Fig. 2, the field immediately preceding the first PIL surface is at a defocused plane, or equivalently, in the near field or Fresnel zone (this correspondence is discussed in detail elsewhere ${ }^{5}$ ). The field, $u_{1}$, at the defocused plane1 is

$$
u_{1}=\mathfrak{I}\left\{A(x, y) e^{i k 9(x, y)}\right\},
$$

where,

$$
\vartheta(x, y)=\varphi+\varphi_{d},
$$

and $\varphi_{d}(x, y)=a_{d}\left(x^{2}+y^{2}\right)$ is the quadratic (defocus) phase term with $a_{d}$ in units of $\lambda$. We note that it is also possible to place $u_{1}$ much farther into the near field, i.e., closer to the aperture stop, by using the Huygens-Fresnel propagator (a derivation in the pupil imaging context is given elsewhere $;^{6} \otimes$ denotes convolution):

$$
u_{1}=\mathfrak{I}\left\{A(x, y) e^{i k \varphi(x, y)}\right\} \otimes e^{i \frac{k}{2 z}\left(x^{2}+y^{2}\right)} .
$$


However, expression (2) is the most relevant for our model since the experimental setup has the PIL placed adjacent from best focus. We make the point that after inclusion of the PIL lens in Fig. 2, the PIL is under-filled, and therefore, the primary mirror remains the aperture stop of the optical system.

To proceed, the total phase delay imparted onto the wavefront upon propagation through the PIL, from Fig. 3 is

$$
\begin{aligned}
\Theta & =n t(x, y)+t_{0}-t(x, y)+\gamma(x, y) \\
& =t_{0}+(n-1) t(x, y)+\gamma(x, y),
\end{aligned}
$$

where $n t(x, y)$ is the phase delay due to the lens with index of refraction, $n$, and $t_{0}-t(x, y)$ is the phase delay due to the intervening space from both the front and back of the lens. The $\gamma(x, y)$ term represents the overall contribution due to the optical path difference as a result of the PIL aberrations. Therefore, the field at surface 2 of the PIL shown in Fig. 2 is given by the product:

$$
u_{2}=u_{1} e^{i k \Theta(x, y)}=u_{1}\left(e^{i k t_{0}}\right)\left(e^{i k(n-1) t(x, y)}\right) e^{i k \gamma(x, y)} .
$$

The first exponential in (6) is a constant; the second term is the phase delay due to the lens with index of refraction, $n$, and the third term is the phase delay due to the lens aberrations.

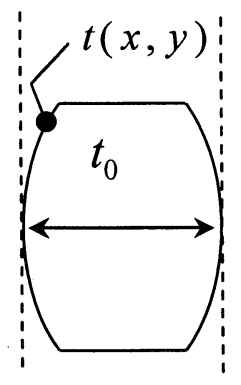

To continue, we must express $t(x, y)$ in terms of the pupil coordinates, $(x, y)$. A standard derivation ${ }^{7}$ follows from the construction of Fig. 3. For completeness, we include a derivation of $t(x, y)$ in the Appendix. Using these results, Equation (B30),

Fig. 3. Phase Delay due to the PIL. $t(x, y)$ is expressed:

$$
t(x, y)=t_{0}+\frac{\left(x^{2}+y^{2}\right)}{2}\left(\frac{1}{R_{2}}-\frac{1}{R_{1}}\right) .
$$

The second exponential term of (6) is thus

$$
i k(n-1) t(x, y)=i k(n-1) t_{0}+i k(n-1)\left(\frac{1}{R_{2}}-\frac{1}{R_{1}}\right) \frac{\left(x^{2}+y^{2}\right)}{2},
$$

but note that the focal length of the lens is given by

$$
\frac{1}{f}=-(n-1)\left(\frac{1}{R_{2}}-\frac{1}{R_{1}}\right),
$$

which allows (8) to be expressed in the simpler form:

$$
i k(n-1) t(x, y)=i k n t_{0}-i k t_{0}-i k \frac{\left(x^{2}+y^{2}\right)}{2 f} .
$$

Therefore, the field at surface 2 of the PIL as shown in Fig. 2 is given by the product:

$$
\begin{aligned}
u_{2} & =u_{1}\left(e^{i k t_{0}}\right)\left(e^{-i k t_{0}}\right)\left(e^{-i k n t_{0}}\right)\left(e^{-i \frac{k}{2 f}\left(x^{2}+y^{2}\right)}\right) e^{i k \gamma(x, y)} \\
& =u_{1}\left(e^{-i k n t_{0}}\right) e^{-i \frac{k}{2 f}\left(x^{2}+y^{2}\right)} e^{i k \gamma(x, y)} .
\end{aligned}
$$

In the back focal plane of the lens the field, $u_{3}$, is obtained by propagation of $u_{2}$ using the Huygens-Fresnel integral:

$$
\begin{aligned}
u_{3} & =u_{2} \otimes\left[e^{i \frac{k}{2 z}\left(x^{2}+y^{2}\right)}\right]_{z \rightarrow f}=\frac{\varepsilon_{A}}{z} e^{i k z} \int_{-\infty}^{\infty} d x^{\prime} d y^{\prime}\left[u_{2}\right] e^{i \frac{k}{2 f}\left[\left(x-x^{\prime}\right)^{2}+\left(y-y^{\prime}\right)^{2}\right]} \\
& =\text { constant } \times \int_{-\infty}^{\infty} d x^{\prime} d y^{\prime}\left[u_{2}\right] e^{i \frac{k}{2 f}\left(x^{\prime 2}+y^{\prime 2}\right)} e^{-i \frac{k}{z}\left(x x^{\prime}+y y^{\prime}\right)}
\end{aligned}
$$

where the second form is obtained after expanding the exponential in the preceding integral. Substituting $u_{2}$ from (11) and noting that in the back focal plane, $z \rightarrow f$, the field at $u_{3}$ is then 


$$
\begin{aligned}
u_{3} & \approx \int_{-\infty}^{\infty} d x^{\prime} d y^{\prime}\left[u_{1}\left(e^{-i k n t_{0}}\right) e^{-i \frac{k}{2 f}\left(x^{2}+y^{2}\right)} e^{i k \gamma(x, y)}\right] e^{i \frac{k}{2 f}\left(x^{\prime 2}+y^{\prime 2}\right)} e^{-i \frac{k}{z}\left(x x^{\prime}+y y^{\prime}\right)} \\
& =e^{-i k n t_{0}} \int_{-\infty}^{\infty} \int_{-\infty} d x^{\prime} d y^{\prime}\left[u_{1} e^{i k \gamma(x, y)}\right] e^{-i \frac{k}{z}\left(x x^{\prime}+y y^{\prime}\right)},
\end{aligned}
$$

where the quadratic phase factors cancel and the constant term, $e^{-i k n t_{0}}$, is factored outside of the integral. Normalizing this result, the field at $u_{3}$ is then expressed:

$$
u_{3}=\int_{-\infty}^{\infty} \int_{-\infty} d x^{\prime} d y^{\prime}\left[u_{1} e^{i k \gamma(x, y)}\right] e^{-i \frac{k}{z}\left(x x^{\prime}+y y^{\prime}\right)},
$$

which is a Fourier transform:

$$
u_{3}=\mathfrak{I}\left\{u_{1} e^{i k \gamma(x, y)}\right\}=\mathfrak{I}\left\{u_{1}\right\} \otimes \mathfrak{I}\left\{e^{i k \gamma(x, y)}\right\},
$$

expressed on the RHS as a convolution product of the Fourier transform of the incident field and the Fourier transform of the PIL complex amplitude defined by the lens aberrations. Substituting from (2) for $u_{1}$ we get the result

$$
u_{3}=\mathfrak{I}\left\{\mathfrak{I}\left[A(x, y) e^{i k \vartheta(x, y)}\right]\right\} \otimes \mathfrak{I}\left\{e^{i k \gamma(x, y)}\right\}
$$

The result in (16) can be further simplified by noting the mathematical identity associated with the Fourier transform:

$$
g(-v)=\mathfrak{I}\{\mathfrak{I}[g(x)]\}
$$

i.e., the Fourier transform of the Fourier transform gives back a scaled and rotated version of the original function. Applying (17) to (16) gives the pupil image complex amplitude at the detector, plane 3:

$$
I_{p u p i l, c a} \equiv u_{3}=\left[A\left(-v_{x},-v_{y}\right) e^{i k \vartheta\left(-v_{x},-v_{y}\right)}\right] \otimes \mathfrak{I}\left\{e^{i k \gamma(x, y)}\right\} \text {. }
$$

The result in (18) states that the pupil image intensity is given by the product of the parent optical system pupil complex amplitude, pupil ${ }_{c a, \text { optical system }}$, convolved with the PIL amplitude spread function, $A S F_{P I L}^{\prime}=\mathfrak{I}\left\{e^{i k \gamma(x, y)}\right\}$, i.e., the PIL amplitude spread function without a PIL aperture response:

$$
I_{\text {pupil, Img }} \equiv I_{\text {pupil }, c a} \cdot \bar{I}_{\text {pupil }, c a}=\left\|p u p i l_{c a} \otimes A S F_{P I L}^{\prime}\right\|^{2} \text {. }
$$

The prime on $A S F_{P I L}^{\prime}$, denotes that it is not the true complex amplitude of the PIL since no aperture term for the PIL is included in the right-hand term of (18). The reason is that the PIL imaging was assumed to be under-filled as it is for the JWST PIL optical design, and the laboratory system we have constructed in Fig. 2. However, there is no more difficulty in allowing the PIL to be overfilled, in this case Equation (18) then becomes:

$$
I_{p u p i l, c a} \equiv u_{3}=\left[A\left(-v_{x},-v_{y}\right) e^{i k \vartheta\left(-v_{x},-v_{y}\right)}\right] \otimes \mathfrak{I}\left\{A_{P I L}(x, y) e^{i k \gamma(x, y)}\right\} \text {. }
$$

An important limit follows from (18) for the case that the PIL lens has no aberrations: the pupil image complex amplitude is simply a scaled version of the complex aperture stop:

$$
I_{\text {pupil }, c a}=A\left(-v_{x},-v_{y}\right) e^{i k \vartheta\left(-v_{x},-v_{y}\right)} .
$$

Therefore, the pupil image collected by the detector at the focal plane follows from the complex conjugate square of (18)

$$
I_{\text {pupil }, c a} \cdot \bar{I}_{\text {pupil,ca }} \Rightarrow I_{\text {pupil,img }}=A\left(-v_{x},-v_{y}\right)^{2}
$$

showing that when no aberrations are introduced by the PIL $(\gamma(x, y)=0)$, the image is essentially the image predicted by geometric optics, i.e., the pupil image is a scaled version of the aperture stop.

This conclusion is essentially a well-known result ${ }^{7}$ (p. 130, Equation (6-3)) and forms the basis of Fourier optics, since this result allows a system to be compressed from a complex multi-plane system to a simple system consisting of entrance and exit pupils and focal planes, i.e., the optical system may be regarded as a black-box. Note especially that for the case of a perfect PIL lens $(\gamma(x, y)=0)$, the primary mirror aberrations, $\varphi(x, y)$ (see Equation (3)), do not affect the pupil image quality. This is due to a cancellation of the complex phase term in the (19). The result is perfectly reasonable to within the paraxial assumption since in essence, the PIL is imaging the primary mirror boundary which is the aperture stop in the system. 


\section{EXAMPLES}

Given the result in (18), it straightforward to calculate the image quality for an arbitrary PIL system given the optical path difference induced by the PIL lens itself, $\gamma(x, y)$, where $\gamma(x, y)$ is available from a ray-trace model or phaseretrieval measurement. ${ }^{3}$ For the system modeled in Fig. 2, our ray-trace model predicts $\gamma(x, y) \approx 1$ wave of spherical aberration where 1 wave $=670 \mathrm{~nm}$. A comparison of both the model and data are shown in Fig. 4 where good qualitative agreement is shown upon comparison of the data and model. No attempt was made to account for detector and sampling effects in the correlation of data and model.

(a)

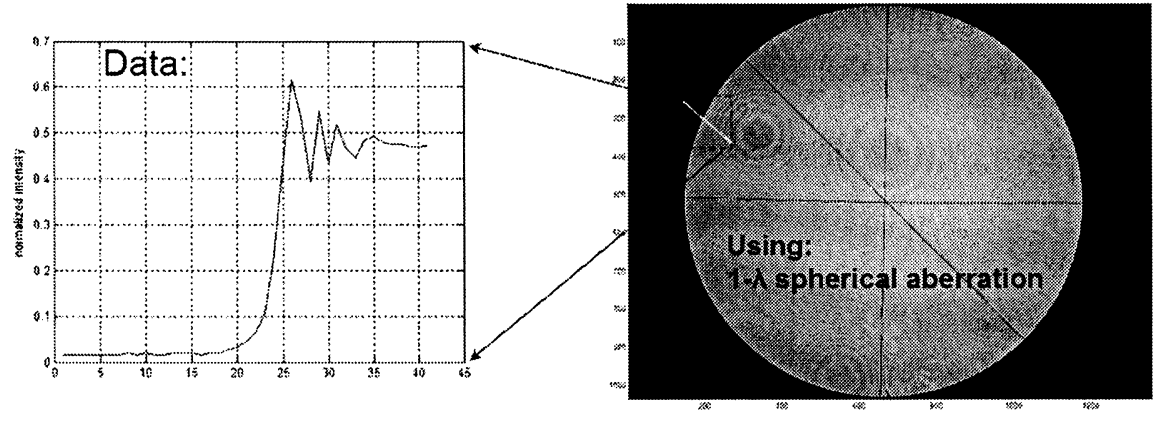

(b)

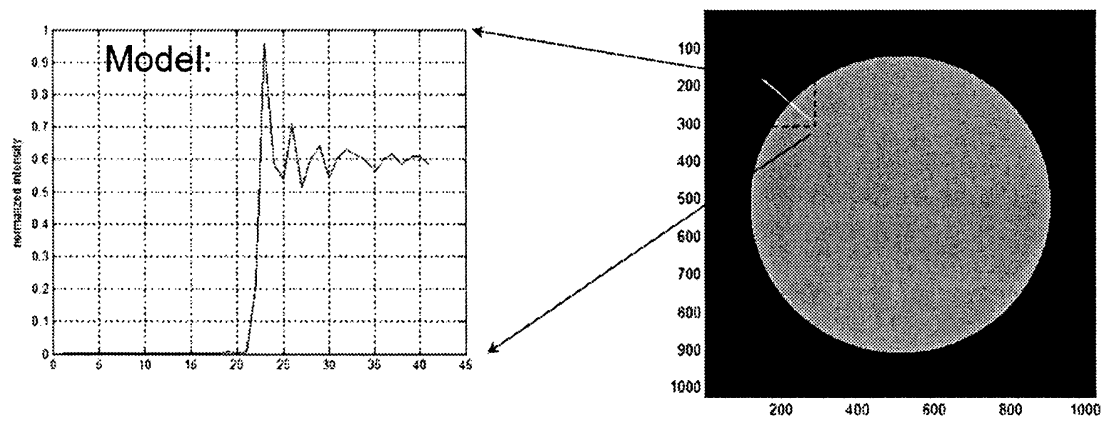

Fig. 4. Comparison of Data (a) and Model (b).

It is important to point out that our coherent imaging model predicts the well-known ringing ${ }^{8}$ in the shadow, although detector sampling in our laboratory measurements did not permit it measurement. This is illustrated across one of the segment edges in the JWST model in Fig. 5.

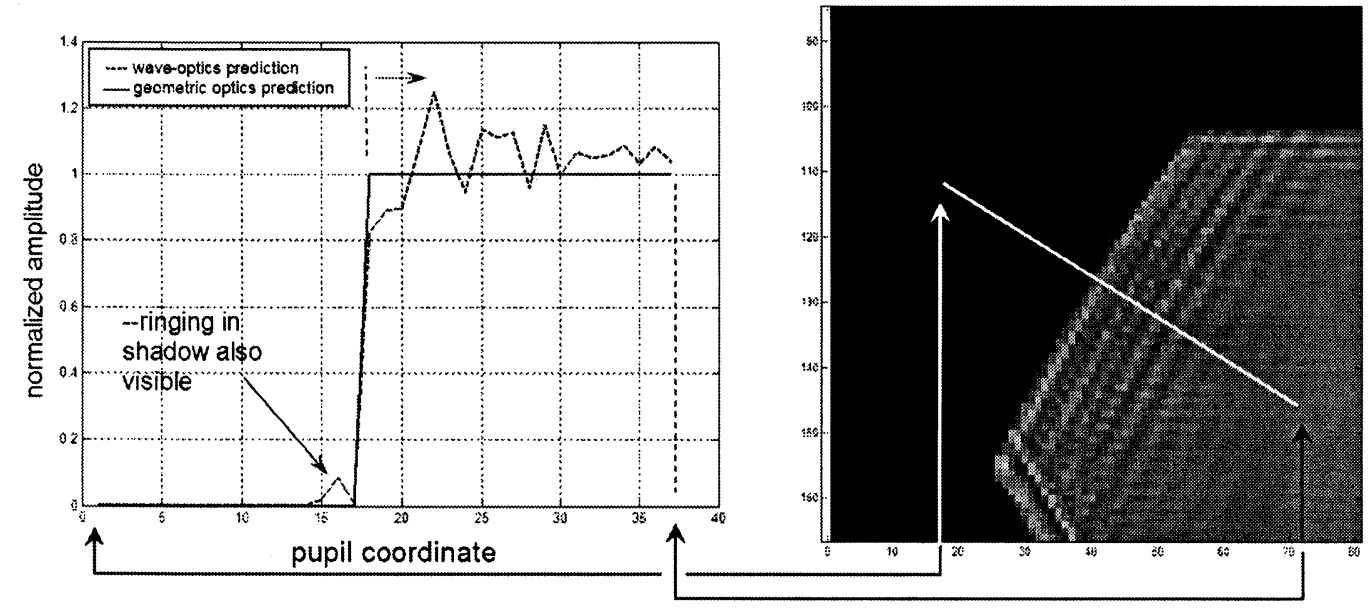

Fig. 5. Coherent ringing across a segment edge in the JWST model. 
As a final application of the wave-optics pupil imaging analysis, we calculate the predicted image-quality of the JWST PIL design. ${ }^{9}$ To determine the in-situ PIL optical path difference, $\gamma(x, y)$, we use a full-ray-trace model of the JWST flight design ${ }^{10}$ including the Near-Infrared Camera (NIRCam) ${ }^{11}$ in combination with the PIL. The $\gamma(x, y)$ is taken to be the difference between the exit pupil optical path difference (OPD), first with the PIL in the design, and then with the PIL removed from the design. The results are shown in Fig. 6 in the Malacara basis set. ${ }^{12}$
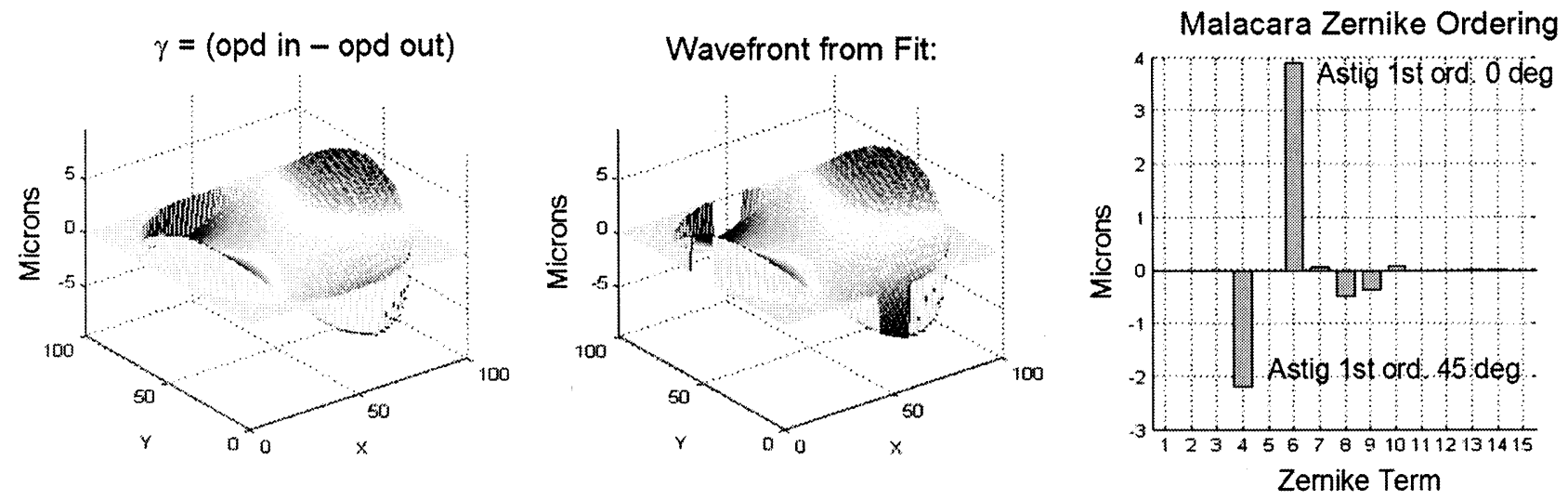

Fig. 6. JWST PIL optical path difference, $\gamma(x, y)$.

The numerical values of the decomposition shown in Fig. 6 are listed in Table 1 in a variety of units.

Table 1. Zernike decomposition of the JWST PIL $\gamma(x, y)$

\begin{tabular}{|c|c|c|c|c|c|c|}
\hline $\begin{array}{c}\mathrm{Z4} \\
\text { Astigmatism } \\
\text { (1st order } \mathbf{4 5}^{\circ} \text { ) } \\
\end{array}$ & $\begin{array}{c}\text { Z6 } \\
\text { Astigmatism } \\
\left(1 \text { st order } 0^{\circ}\right)\end{array}$ & $\begin{array}{c}\mathbf{Z 7} \\
\text { Trefoil 30 }\end{array}$ & $\begin{array}{c}\mathrm{Z8} \\
\text { Coma Y }\end{array}$ & $\begin{array}{c}\mathrm{Z9} \\
\text { Coma X }\end{array}$ & $\begin{array}{c}\mathrm{Z10} \\
\text { Trefoil } 0^{\circ}\end{array}$ & UNITS \\
\hline-2.1715 & 3.9015 & 0.0594 & -0.4861 & -0.3603 & 0.0961 & $\mu m$ \\
\hline-1.1524 & 2.0705 & 0.0315 & -0.2580 & -0.1912 & 0.0510 & $\begin{array}{l}\text { waves }(\lambda= \\
1.884 \mu \mathrm{m})\end{array}$ \\
\hline-2171 & 3901 & 59.42 & -486.15 & -360.27 & 96.06 & nanometers \\
\hline
\end{tabular}

As shown in Table 1 and Fig. 6, the dominant contributor to $\gamma(x, y)$ is astigmatism. Substituting $\gamma(x, y)$ into $(18)$ and then using the 18-segment mask of the JWST design for $A\left(-v_{x},-v_{y}\right)$, a "cross-stitch" ringing pattern is induced from the astigmatic nature of $\gamma(x, y)$. The result is shown in Fig. 9. The structure of this astigmatic ringing pattern is better understood by using a circular aperture mask for the aperture stop, but with the same $\gamma(x, y)$. This result is shown in Fig. 8, where it is apparent that the astigmatism introduces the rather unusual "cross-stitch" ringing pattern.

\section{SUMMARY AND DISCUSSION}

We have presented a paraxial wave-optics model of coherent pupil imaging and have compared modeling results with pupil images collected in the laboratory. An interesting result is that coherent ringing may be traced to the PIL lens aberrations, or optical path delay. Indeed, for the limiting case that $\gamma(x, y)=0$, the resulting image produced by the PIL is essentially the image predicted by geometric optics, i.e., the pupil image is a scaled version of the aperture stop.

The practical implications are that the pupil image will not be significantly degraded by diffraction effects if two conditions are met: optical aberrations are well-corrected and the subsequent optical system apertures do not clip the wavefront coming from the mirror's surface, recalling the assumption that the PIL lens itself was assumed to underfilled. ${ }^{13}$ Therefore, the pupil image that is produced from a well-corrected PIL with sufficiently oversized apertures will closely approximate the pupil image predicted by geometrical optics, for obscurations located on or near to the system aperture stop. 
The experimental setup described in this paper, although generic for pupil imaging systems in general, also has application to the James Webb Space Telescope (JWST) optical system characterization where the pupil images are used as a constraint to the wavefront sensing and control process. We have determined the in-situ JWST PIL optical path difference using the flight ray-trace model and have used these results to predict the exact nature of the coherent ringing produced by the PIL imaging system. An obvious concern due to the ringing in the PIL images is the ability to resolve or register pupil edges. Although is not necessarily a concern for systems with a simple circular aperture stop, this is definitely a concern for complex apertures such as the JWST 18 segment aperture. We note however that using a straightforward image processing technique such as deconvolution can reduce these ringing effects. An example of this post-processing step is shown in Fig. 7.

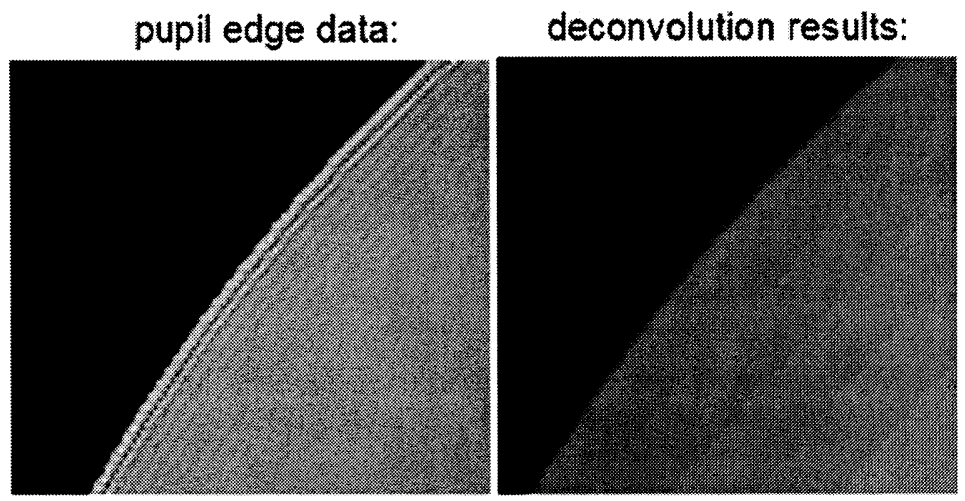

Fig. 7. Pupil image coherent ringing reduction using one deconvolution iteration.

A much simpler and more effective technique for reducing the edge ringing is to image the pupil in broadband light. In this case the temporal coherence length, $L_{c}:{ }^{14}$

$$
L_{c}=\lambda^{2} / \Delta \lambda,
$$

tends to zero and the coherent ringing disappears from the images. Given that the signal-noise-ratio of the broadband light will be greater than in the narrow-band case, the resulting pupil imaging exposure times can be significantly reduced as well, at the expense of additional chromatic imaging effects.

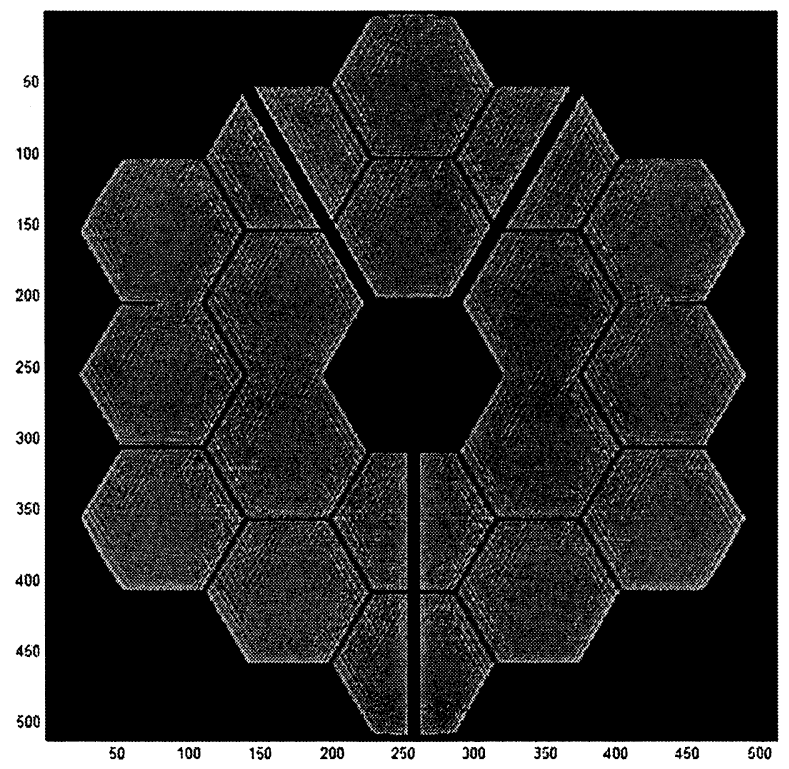

Fig. 8. Image Predicted by Coherent Model of JWST Pupil Imaging. 


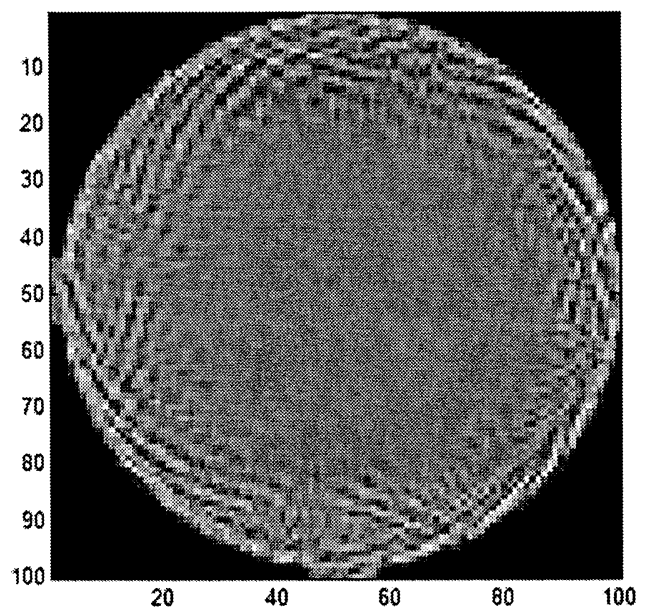

Fig. 9. Image Predicted by Coherent Model using a Circular Pupil with the JWST PIL $\gamma(x, y)$.

\section{APPENDIX}

For completeness, a derivation of $t(x, y)$ is given. Other derivations may be found in the literature. ${ }^{7}$ To begin, we adopt the convention shown in Fig. 10(a) that the radius of curvature is positive for the first surface (convex) and that $R<0$ for the second surface (concave). To find the explicit form of $t(x, y)$ in terms of the pupil coordinates, $(x, y)$, the geometry of Fig. 3 is further divided into three regions as shown in Fig. 10(b).
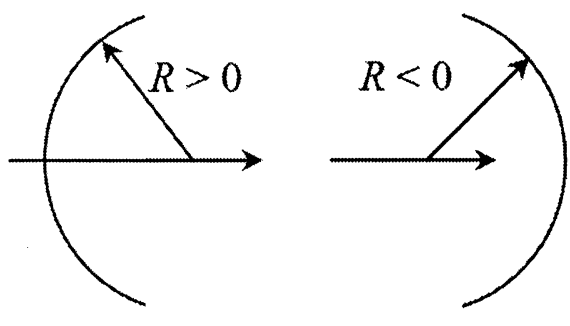

(a)

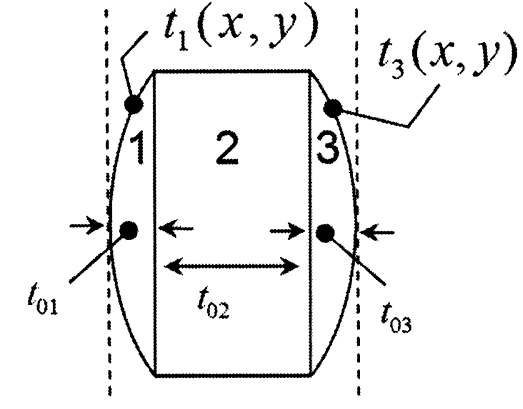

(b)

Fig. 10. (a) Radius of Curvature Sign Convention; (b) Regions of Phase Delay due to the PIL.

Therefore, we consider

$$
t(x, y)=t_{1}+t_{2}+t_{3}
$$

and

$$
t_{0}=t_{01}+t_{02}+t_{03}
$$

where $t_{0}$ defines the thickness of the lens long the optical axis. Fig. 11 shows additional detail on Region 1 of Fig. 10(b). 


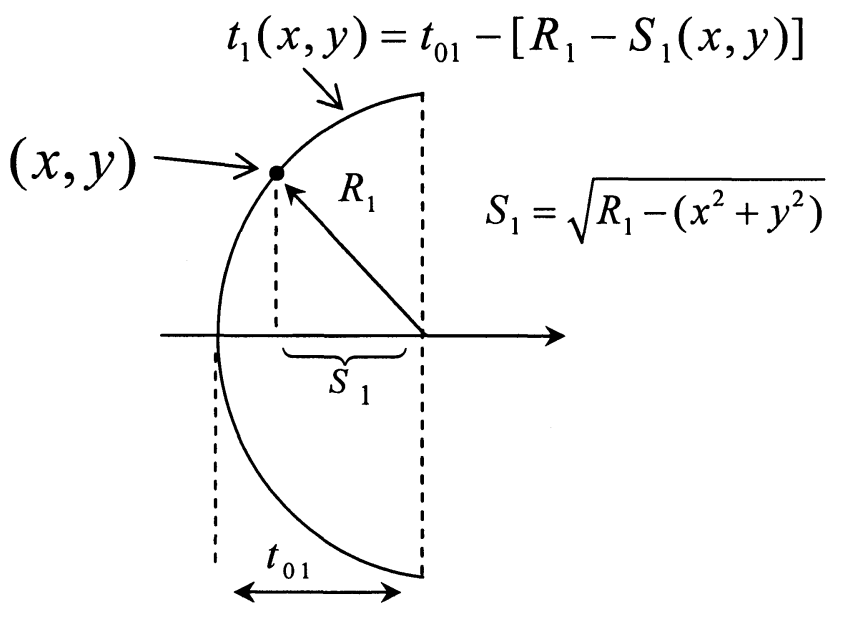

Fig. 11. Detail on Region 1.

From Fig. 11 the explicit form of $t_{1}(x, y)$ is obtained:

$$
\begin{aligned}
t_{1}(x, y) & =t_{01}-\left[R_{1}-S_{1}(x, y)\right] \\
& =t_{01}-\left[R_{1}-\sqrt{R_{1}-\left(x^{2}+y^{2}\right)}\right] \\
& =t_{01}-R_{1}\left[1-\sqrt{1-\left(x^{2}+y^{2}\right) / R_{1}^{2}}\right] .
\end{aligned}
$$

The expression for $t_{1}(x, y)$ is further simplified using the binomial approximation which is equivalent to the small angle approximation of paraxial optics:

$$
\begin{aligned}
t_{1}(x, y) & =t_{01}-R_{1}\left[1-\left(1-\left(x^{2}+y^{2}\right) / 2 R_{1}^{2}\right)\right] \\
& \left.=t_{01}-R_{1}\left[\left(x^{2}+y^{2}\right) / 2 R_{1}^{2}\right)\right] \\
& =t_{01}-\left(x^{2}+y^{2}\right) / 2 R_{1} .
\end{aligned}
$$

The expression for $t_{2}(x, y)$ is similarly obtained:

$$
t_{2}(x, y)=t_{02}+\left(x^{2}+y^{2}\right) / 2 R_{2},
$$

after factoring out a $(-1) R_{2}$ term consistent with the sign convention of Fig. 10. Substituting (B27) and (B28) into (B24) and then using the fact that $t_{2}=t_{02}$ gives

$$
t(x, y)=t_{01}+t_{02}+t_{03}+\frac{\left(x^{2}+y^{2}\right)}{2}\left(\frac{1}{R_{2}}-\frac{1}{R_{1}}\right),
$$

and after using (B25) the result simplifies to

$$
t(x, y)=t_{0}+\frac{\left(x^{2}+y^{2}\right)}{2}\left(\frac{1}{R_{2}}-\frac{1}{R_{1}}\right) .
$$




\section{ACKNOWLEDGEMENTS}

The research described in this paper was carried out at the NASA Goddard Space Flight Center. The authors acknowledge support from the JWST project office and helpful discussion with Joe Howard, Bill Hayden, Erin Sabatke, Scott Acton, and members of the Lockheed Martin and University of Arizona NIRCam Team.

\section{REFERENCES}

1. results in this paper are based on:

B. H. Dean, "Wave-Optics Analysis of Pupil Imaging," JWST Technical Memoranda, Sept 28 (2005).

B. H. Dean, B. Bos, and J. Howard, "Wave-Optics Analysis of JWST Pupil Imaging," JWST Technical Memoranda, Nov 9 (2005).

- in response to questions originally posed by Don Davies and Bill Hayden.

2. P.A. Sabelhaus, D. Campbell, M. Clampin, J. Decker, M. Greenhouse, A. Johns, M. Menzel, R.Smith, P. Sullivan, "An overview of the James Webb Space Telescope (JWST) project," Proceedings of SPIE, Vol. 5899 (2005).

C. B. Atkinson, S. C. Texter, L. D. Feinberg, R. A. M. Keski-Kuha "Status of the JWST optical telescope element," these proceedings, SPIE Orlando (2006).

B. J. Bos, P. S. Davila, M. Jurotich, G. Hobbs, P. Lightsey, J. Contreras and T. Whitman, The James Webb Space Telescope instrument suite layout: optical system engineering considerations for a large, deployable space telescope, Proc. of SPIE, 5487 (2004).

3. B. H. Dean, D. S. Acton, D. Aronstein, S. Smith, and R. Shiri, "Phase-Retrieval Algorithm for JWST Flight and Testbed Telescope," Proc. of SPIE, these proceedings, Orlando (2006).

4. Edmund Scientific: http://www.edmundoptics.com/

5. B. H. Dean, C. Bowers, "Diversity Selection for Phase-Diverse-Phase-Retrieval," J. Opt. Soc. Am. 20, 14901504 (2003).

6. B. Dean, "Computational Optics in the Near Field: Extended Range for JWST WFS," JWST WFS\&C Working Group, presented at JPL, Feb. 24 (2005).

7. J. W. Goodman, Introduction to Fourier Optics, $2^{\text {nd }}$ edn. (McGraw Hill, New York, NY, 1996).

8. for comparison, see p. 159 , Figure 6.20 , of reference, ${ }^{7}$

9. C. S. Clark and T. Jamieson, "NIRCam pupil imaging lens mechanism and optical design," Proc. of SPIE, Vol. 5904 (2005).

10. J. W. Contreras and P. A. Lightsey, Optical design and analysis of the James Webb Space Telescope: optical telescope element, Proceedings of SPIE, Vol. 5524, 30 (2004).

11. L. A. Ryder and Thomas Jamieson, "Lens design for the Near Infrared Camera for the James Webb Space Telescope," Proc. SPIE 5904 (2005). 
L. G. Burriesci, "NIRCam instrument overview," Proceedings of SPIE, Vol. 5904, 590403 (2005).

M. J. Rieke and R. C. Powell, "James Webb Space Telescope Program NIRCam Concept Study Report," Univ of Arizona, Jan 31 (2003).

Additional NIRCam information is available on the website: http://ircamera.as.arizona.edu/nircam/

12. D. Malacara and S DeVore, "Interferogram Evaluation and Wavefront Fitting," in Optical Shop Testing, Wiley Series in Pure and Applied Optics, NY (1997).

13. A generalization to this result is obtained by assuming that the PIL lens is over-filled by the beam at position (1) of Fig. 2. This analysis will be documented elsewhere.

14. Reynolds, DeVelis, Parrent, Thompson, "New Physical Optics Notebook," SPIE Optical Engineering Press, NY, 1989. 